\title{
Rigid Body Time Integration by Convected Base Vectors with Implicit Constraints
}

\author{
Krenk, Steen; Nielsen, Martin Bjerre
}

Published in:

COMPDYN 2013. 4th ECCOMAS Thematic Conference on Computational Methods in Structural Dynamics and Earthquake Engineering

Publication date:

2013

Link back to DTU Orbit

Citation (APA):

Krenk, S., \& Nielsen, M. B. (2013). Rigid Body Time Integration by Convected Base Vectors with Implicit Constraints. In M. Papadrakakis, V. Papadopoulos, \& V. Plevris (Eds.), COMPDYN 2013. 4th ECCOMAS Thematic Conference on Computational Methods in Structural Dynamics and Earthquake Engineering

\section{General rights}

Copyright and moral rights for the publications made accessible in the public portal are retained by the authors and/or other copyright owners and it is a condition of accessing publications that users recognise and abide by the legal requirements associated with these rights.

- Users may download and print one copy of any publication from the public portal for the purpose of private study or research.

- You may not further distribute the material or use it for any profit-making activity or commercial gain

- You may freely distribute the URL identifying the publication in the public portal 


\title{
RIGID BODY TIME INTEGRATION BY CONVECTED BASE VECTORS WITH IMPLICIT CONSTRAINTS
}

\author{
S. Krenk and M.B. Nielsen \\ Department of Mechanical Engineering \\ Technical University of Denmark \\ DK-2800 Kgs. Lyngby, Denmark \\ e-mail: sk@mek.dtu.dk,mbni@mek.dtu.dk
}

Keywords: Conservative Time Integration, Rigid Body Rotations, Implicit Constraints, Structural Dynamics.

\begin{abstract}
A conservative time integration algorithm based on a convected set of orthonormal base vectors is presented. The equations of motion are derived from an extended Hamiltonian formulation, combining the components of the three base vectors with a set of orthonormality constraints. The particular form of the kinetic energy used in the present formulation is deliberately chosen to correspond to a rigid body rotation, and the orthonormality constraints are introduced via the equivalent Green strain components of the base vectors. The particular form of the extended inertia tensor used here implies a set of orthogonality relations between the base vector components and their conjugate momentum components. These orthogonality relations permit explicit elimination of the Lagrange multipliers associated with the constraints, leading to a projected form of the dynamic equation without explicit algebraic constraints. The differential equations of motion are recast into discrete form using a suitable combination of mean values and increments, which is identified by considering a finite increment of the Hamiltonian. Examples illustrate the accuracy and conservation properties of the algorithm.
\end{abstract}




\section{INTRODUCTION}

Numerical time integration of the motion of rigid bodies has been subject to intensive studies. In [1] a new approach to time integration of rigid body motion was introduced in which energy and momentum conservation properties are obtained by judicious discretization in terms of mean values and increments. In contrast to earlier methods based on asymptotic properties, the conservative algorithms depend in an essential way on the parameter representation of the problem. A fully conservative algorithm in terms of quaternion parameters can be obtained when the normalization condition is carried through the integration process via a Lagrange multiplier [2]. It was demonstrated in [3] that the rigid body dynamics problem can be formulated in such a way that the increment of the constraint is embedded in the kinematic evolution equation, and the Lagrange multiplier can be eliminated, leading to the introduction of a projection operator on the force potential gradient. An alternative formulation of the rigid body motion in terms of a set of convected base vectors has been introduced in [4]. The use of the global components of the local base vectors as variables simplifies the formulation, but at the same time increases the problem size and changes the character of the problem by adding 6 Lagrange multipliers and introducing algebraic constraints.

In the present paper this problem is solved by extending the idea of 'implicit constraints' introduced in [3] to the formulation in terms of convected base vectors. The equations of motion are obtained from Hamilton's equations. It turns out, that when the kinetic energy is formulated via the angular velocity components under the assumption of a rigid body, the rigid body constraints are in fact contained in incremental form in the set of kinematic Hamilton equations. The generalized forces appear in the dynamic part of the Hamilton equations in the form of the gradient of the force potential. The special form of the inertial tensor in terms of the base vector components leads to a set of six orthogonality conditions between the base vector components and the corresponding momentum components. Elimination of the Lagrange multipliers by use of these orthogonality relations leads to a set of equations of motion, in which the effect of the constraints is represented via pre-multiplication of the full-component gradient by a projection matrix.

The modified Hamilton equations are discretized by appropriate mean values and increments to form an energy and momentum conserving time integration algorithm. The accuracy and conservation properties are illustrated by the 'flying brick' and the rotation of a Lagrangian top in a gravitational field.

\section{CONVECTED BASE VECTOR REPRESENTATION}

Let $\mathbf{x}$ denote the location of a rigid body in a fixed global frame of reference and let a local frame attached to the rigid body be described in terms of a set of orthonormal base vectors $\mathbf{q}_{1}, \mathbf{q}_{2}, \mathbf{q}_{3}$. For simplicity, the present formulation is developed for purely rotational motion where the origin of the local base coincides with the origin of the global frame, hence the global components $\mathbf{x}$ of a point inside the body with local coordinates $\mathbf{x}_{0}$ can be expressed as

$$
\mathbf{x}(t)=\mathbf{Q}(t) \mathbf{x}_{0},
$$

where the deformation gradient tensor $\mathbf{Q}$ is defined as

$$
\mathbf{Q}=\left[\mathbf{q}_{1}, \mathbf{q}_{2}, \mathbf{q}_{3}\right]=\frac{\partial \mathbf{x}}{\partial \mathbf{x}_{0}} .
$$

The global components of the base vectors $\mathbf{q}_{1}, \mathbf{q}_{2}, \mathbf{q}_{3}$ constitute the independent variables of the present formulation. However, in order to represent a proper rigid body rotation the base vectors 
must remain orthonormal at all time. This corresponds to vanishing of all Green strain components, which can be expressed by a set of constraint conditions on the deformation gradient tensor $\mathbf{Q}$ as

$$
\mathbf{e}=\frac{1}{2}\left[\begin{array}{c}
\mathbf{q}_{1}^{T} \mathbf{q}_{1}-1 \\
\mathbf{q}_{2}^{T} \mathbf{q}_{2}-1 \\
\mathbf{q}_{3}^{T} \mathbf{q}_{3}-1 \\
\mathbf{q}_{2}^{T} \mathbf{q}_{3}+\mathbf{q}_{3}^{T} \mathbf{q}_{2} \\
\mathbf{q}_{3}^{T} \mathbf{q}_{1}+\mathbf{q}_{1}^{T} \mathbf{q}_{3} \\
\mathbf{q}_{1}^{T} \mathbf{q}_{2}+\mathbf{q}_{2}^{T} \mathbf{q}_{1}
\end{array}\right]=\mathbf{0 .}
$$

In the present formulation the kinematic constraints appear via their time derivatives in the form

$$
\dot{\mathbf{e}}=\mathbf{C}(\mathbf{q}) \dot{\mathbf{q}}=\mathbf{C}(\dot{\mathbf{q}}) \mathbf{q}=\mathbf{0},
$$

where the matrix $\mathbf{C}$ follows from differentiation of (3) with the $6 \times 9$ deformation gradient matrix

$$
\mathbf{C}(\mathbf{q})=\frac{\partial \mathbf{e}}{\partial \mathbf{q}}=\left[\begin{array}{ccc}
\mathbf{q}_{1}^{T} & \mathbf{0} & \mathbf{0} \\
\mathbf{0} & \mathbf{q}_{2}^{T} & \mathbf{0} \\
\mathbf{0} & \mathbf{0} & \mathbf{q}_{3}^{T} \\
\mathbf{0} & \mathbf{q}_{3}^{T} & \mathbf{q}_{2}^{T} \\
\mathbf{q}_{3}^{T} & \mathbf{0} & \mathbf{q}_{1}^{T} \\
\mathbf{q}_{2}^{T} & \mathbf{q}_{1}^{T} & \mathbf{0}
\end{array}\right] .
$$

The kinetic energy of a rigid body rotating with angular velocity $\boldsymbol{\omega}$ takes the following form when expressed in local components

$$
T=\frac{1}{2} \boldsymbol{\omega}^{T} \mathbf{J} \boldsymbol{\omega},
$$

where $\mathbf{J}$ is the constant inertia tensor. The local components of the angular velocity in terms of the base vectors is obtained by projecting the derivatives $\dot{\mathbf{q}}_{i}$ on the base vectors $\mathbf{q}_{j}$. This can be arranged into the compact matrix form

$$
\boldsymbol{\omega}=-\frac{1}{2} \mathbf{G}(\mathbf{q}) \dot{\mathbf{q}}
$$

in terms of the $3 \times 9$ matrix

$$
\mathbf{G}(\mathbf{q})=\left[\begin{array}{rrr}
\mathbf{0} & -\mathbf{q}_{3}^{T} & \mathbf{q}_{2}^{T} \\
\mathbf{q}_{3}^{T} & \mathbf{0} & -\mathbf{q}_{1}^{T} \\
-\mathbf{q}_{2}^{T} & \mathbf{q}_{1}^{T} & \mathbf{0}
\end{array}\right]
$$

This matrix $\mathbf{G}(\mathbf{q})$ has the same structure in terms of the base vectors $\mathbf{q}_{1}, \mathbf{q}_{2}, \mathbf{q}_{3}$ as the $3 \times 3$ skewsymmetric matrix associated with the standard vector product, and thus the matrix structure itself implies orthogonality with respect to $\mathbf{q}$ in the sense

$$
\underset{3 \times 9}{\mathbf{G}(\mathbf{q}) \mathbf{q}}=\underset{3 \times 1}{\mathbf{0}} .
$$

Furthermore, it is an important property in the present formulation that $\mathbf{G}(\mathbf{q})$ satisfy the following relation with respect to the constraint matrix $\mathbf{C}(\mathbf{q})$ when the vectors $\mathbf{q}_{j}$ constitute an orthonormal base,

$$
\underset{6 \times 9}{\mathbf{C}(\mathbf{q})} \underset{9 \times 3}{\mathbf{G}(\mathbf{q})^{T}}=\underset{6 \times 3}{\mathbf{0}} .
$$


Upon substitution of the expression for the angular velocity from (7) into (6), the kinetic energy takes the following bi-quadratic form

$$
T=\frac{1}{8} \dot{\mathbf{q}}^{T} \mathbf{G}(\mathbf{q})^{T} \mathbf{J} \mathbf{G}(\mathbf{q}) \dot{\mathbf{q}},
$$

when the base vectors $\mathbf{q}_{j}$ satisfy the constraint (3).

\section{EQUATIONS OF RIGID BODY MOTION}

The equations of motion for rigid body rotation are developed via the Hamilton's canonical equations leading to a set of first order differential equations for the generalized displacements, here represented as the base vector components $\mathbf{q}_{j}$, and their generalized momentum variables, see e.g. [5].

\subsection{Hamilton's equations}

The vector of generalized momentum components $\mathbf{p}=\left[\mathbf{p}_{1}^{T}, \mathbf{p}_{2}^{T}, \mathbf{p}_{3}^{T}\right]^{T}$ conjugate to the base vectors $\mathbf{q}=\left[\mathbf{q}_{1}^{T}, \mathbf{q}_{2}^{T}, \mathbf{q}_{3}^{T}\right]^{T}$ follows from time differentiation of the kinetic energy (11), as

$$
\mathbf{p}=\frac{\partial T}{\partial \dot{\mathbf{q}}^{T}}=\frac{1}{4} \mathbf{G}(\mathbf{q})^{T} \mathbf{J} \mathbf{G}(\mathbf{q}) \dot{\mathbf{q}}
$$

This gives the relation between the momentum $\mathbf{p}$ and the generalized velocity $\dot{\mathbf{q}}$. For a rigid body the base vectors $\mathbf{q}_{j}$ are orthonormal, and thus the relation (12) can be used to eliminate the velocity $\dot{\mathbf{q}}$ from the kinetic energy when pre-multiplicated with $\mathbf{J}^{-1} \mathbf{G}(\mathbf{q})$. Hereby the kinetic energy can be expressed in either of the forms

$$
T(\mathbf{q}, \mathbf{p})=\frac{1}{2} \mathbf{p}^{T} \mathbf{G}(\mathbf{q})^{T} \mathbf{J}^{-1} \mathbf{G}(\mathbf{q}) \mathbf{p}=\frac{1}{2} \mathbf{q}^{T} \mathbf{G}(\mathbf{p})^{T} \mathbf{J}^{-1} \mathbf{G}(\mathbf{p}) \mathbf{q},
$$

where the latter expression is valid, since the structure of $\mathbf{G}$ ensures that simultaneous interchange of $\mathbf{q}$ and $\mathbf{p}$ in the factors appearing on each side of $\mathbf{J}$ does not change the value of the product.

The present formulation is based on an augmented form of the Hamiltonian where the sum of the kinetic energy $T(\mathbf{q}, \mathbf{p})$ from (13) and the potential energy function $V(\mathbf{q})$ is supplemented by the homogeneous rigid body constraints (3), whereby

$$
H(\mathbf{q}, \mathbf{p})=T(\mathbf{q}, \mathbf{p})+V(\mathbf{q})-\mathbf{e}(\mathbf{q})^{T} \boldsymbol{\lambda} .
$$

The constraints $\mathbf{e}(\mathbf{q})=\mathbf{0}$ are initially introduced via a vector of Lagrange multipliers $\boldsymbol{\lambda}$. They are eliminated subsequently by a displacement-momentum orthogonality relation discussed in the following section.

The equations of motion follow from the augmented Hamiltonian (14) by differentiation, as

$$
\begin{aligned}
& \dot{\mathbf{q}}=\frac{\partial H}{\partial \mathbf{p}^{T}}=\mathbf{G}(\mathbf{q})^{T} \mathbf{J}^{-1} \mathbf{G}(\mathbf{q}) \mathbf{p} \\
& \dot{\mathbf{p}}=-\frac{\partial H}{\partial \mathbf{q}^{T}}=-\mathbf{G}(\mathbf{p})^{T} \mathbf{J}^{-1} \mathbf{G}(\mathbf{p}) \mathbf{q}-\frac{\partial V}{\partial \mathbf{q}^{T}}+\mathbf{C}(\mathbf{q})^{T} \boldsymbol{\lambda}
\end{aligned}
$$

where the derivatives of the kinetic energy follow from (13), and the deformation gradient $\mathbf{C}(\mathbf{q})$ is given by (5). 


\subsection{Elimination of Lagrange multipliers}

A basic feature of the present formulation is the elimination of the Lagrange multipliers by use of a set of orthogonality conditions between the generalized displacements $\mathbf{q}$ and the conjugate momentum vector $\mathbf{p}$. The orthogonality relations are obtained by pre-multiplication of the relation (12) defining $\mathbf{p}$ with $\mathbf{C}(\mathbf{q})$. When using the relation (10), valid for orthogonal base vectors, the following displacement-momentum relation is obtained,

$$
\mathbf{C}(\mathbf{q}) \mathbf{p}=\mathbf{0}
$$

In spite of the resemblance of this relation to the velocity relation (4), it is not a simple reformulation of the constraint derivative conditions.

The Lagrange multipliers are now eliminated by using the time derivative of the displacementmomentum orthogonality relations (17),

$$
\mathbf{C}(\mathbf{p}) \dot{\mathbf{q}}+\mathbf{C}(\mathbf{q}) \dot{\mathbf{p}}=\mathbf{0} .
$$

Substitution of the time derivatives from (15) and (16) into this equation leads to the following expression for the Lagrange multiplier vector

$$
\boldsymbol{\lambda}=\left[\mathbf{C}(\mathbf{q}) \mathbf{C}(\mathbf{q})^{T}\right]^{-1} \mathbf{C}(\mathbf{q}) \frac{\partial V}{\partial \mathbf{q}^{T}} .
$$

In particular, it is noticed that the Lagrange multipliers vanish in the absence of external loads, implying that the homogenous equations with the present form of the inertial matrix can be solved without explicit introduction of constraints. Substitution of the Lagrange multiplier vector (19) into the dynamic equation of motion (16) gives

$$
\dot{\mathbf{p}}=-\mathbf{G}(\mathbf{p})^{T} \mathbf{J}^{-1} \mathbf{G}(\mathbf{p}) \mathbf{q}-\left(\mathbf{I}-\mathbf{C}(\mathbf{q})^{T}\left[\mathbf{C}(\mathbf{q}) \mathbf{C}(\mathbf{q})^{T}\right]^{-1} \mathbf{C}(\mathbf{q})\right) \frac{\partial V}{\partial \mathbf{q}^{T}} .
$$

It is seen that the elimination of the Lagrange multipliers via the constraint derivative is equivalent to subtracting the projection of the external potential gradient on the deformation modes via $\mathbf{C}(\mathbf{q})$ from the unconstrained gradient, leaving only the components associated with the rotation modes. This is similar to the result in [3] when eliminating the single scalar normalization constraint from the four-component quaternion representation of rigid-body rotation.

\section{STATE-SPACE TIME INTEGRATION}

The basic idea of conservative time integration is to use an integrated form of the evolution equations. Hereby the discretized form of the equations of motion can be designed to yield the correct incremental change of energy and momentum over a finite time interval $\Delta t$ from $t_{n}$ to $t_{n+1}$. This is different from collocation based methods where the equations of motion are matched at discrete points in time. Similarly, when constraints are included in conservative methods via Lagrange multipliers, the role of the Lagrange multipliers is to ensure satisfaction of the integrated form of the constraints constraints over the current interval, and thus these are associated with interval rather than the end points. When a numerical time integration is initiated from a state that satisfies the constraints, the introduction of the corresponding incremental form of the constraints over the integration time intervals will lead to satisfaction of the constraints at the integration times. 
A consistent discretization obeying conservation of energy and momentum can be derived by equating the finite increment of the Hamiltonian to zero, as

$$
\Delta H(\mathbf{q}, \mathbf{p})=\Delta \mathbf{q}^{T} \frac{\partial H_{*}}{\partial \mathbf{q}^{T}}+\Delta \mathbf{p}^{T} \frac{\partial H_{*}}{\partial \mathbf{p}^{T}}=0
$$

where the asterisk denotes the finite derivatives of $H$ corresponding to the increments $\Delta \mathbf{q}^{T}$ and $\Delta \mathbf{p}^{T}$. The individual terms can be identified from (14). The kinetic energy is a biquadratic form in $\mathbf{q}$ and $\mathbf{p}$, hence the increment can be expressed as twice the product of one factor plus the increment of the other factor. The potential $V(\mathbf{q})$ is introduced via of its finite derivative $\partial V_{*} / \partial \mathbf{q}$, see e.g. [6], while the discrete form of the constraints follows from the increment of (3). This is a homogeneous quadratic form in $\mathbf{q}$ and can be represented by a combination of increments and mean values. The role of the Lagrange multipliers $\lambda$ is to prevent violation of the internal constraints at the end of the interval when these are satisfied initially, hence these are introduced as effective mean values representing their role over the interval. Hereby the discretized equations of motion take the form

$$
\begin{aligned}
& \Delta \mathbf{q}=\frac{\partial H_{*}}{\partial \mathbf{p}^{T}}=\Delta t \mathbf{G}(\overline{\mathbf{q}})^{T} \mathbf{J}^{-1} \overline{\mathbf{G}(\mathbf{q}) \mathbf{p}}, \\
& \Delta \mathbf{p}=-\frac{\partial H_{*}}{\partial \mathbf{q}^{T}}=-\Delta t \mathbf{G}(\overline{\mathbf{p}})^{T} \mathbf{J}^{-1} \overline{\mathbf{G}(\mathbf{p}) \mathbf{q}}-\Delta t\left[\frac{\partial V_{*}}{\partial \mathbf{q}^{T}}-\mathbf{C}(\overline{\mathbf{q}})^{T} \boldsymbol{\lambda}\right],
\end{aligned}
$$

These equations constitute a clear equivalent to the continuous evolution equations (15) and (16), when the respective gradients are introduced via their finite derivatives.

As in the continuous case it is advantageous to eliminate the explicit dependence of the Lagrange multiplier using the incremental form of the orthogonality relation between displacement and momentum (17),

$$
\mathbf{C}(\overline{\mathbf{p}}) \Delta \mathbf{q}+\mathbf{C}(\overline{\mathbf{q}}) \Delta \mathbf{p}=\mathbf{0} .
$$

Upon insertion of the increments from (22) and (23), solving for the Lagrange multiplier $\boldsymbol{\lambda}$ and back-substitution into (23), the dynamic equation take the form

$$
\begin{aligned}
\Delta \mathbf{p}= & -\Delta t \mathbf{G}(\overline{\mathbf{p}})^{T} \mathbf{J}^{-1} \overline{\mathbf{G}(\mathbf{p}) \mathbf{q}}-\Delta t\left(\mathbf{I}-\mathbf{C}(\overline{\mathbf{q}})^{T}\left[\mathbf{C}(\overline{\mathbf{q}}) \mathbf{C}(\overline{\mathbf{q}})^{T}\right]^{-1} \mathbf{C}(\overline{\mathbf{q}})\right) \frac{\partial V_{*}}{\partial \mathbf{q}^{T}} \\
& +\Delta t \mathbf{C}(\overline{\mathbf{q}})^{T} \boldsymbol{\lambda}_{0} .
\end{aligned}
$$

with

$$
\boldsymbol{\lambda}_{0}=\left[\mathbf{C}(\overline{\mathbf{q}}) \mathbf{C}(\overline{\mathbf{q}})^{T}\right]^{-1}\left[\mathbf{C}(\overline{\mathbf{q}}) \mathbf{G}(\overline{\mathbf{p}})^{T}+\mathbf{C}(\overline{\mathbf{p}}) \mathbf{G}(\overline{\mathbf{q}})^{T}\right] \mathbf{J}^{-1} \overline{\mathbf{G}(\mathbf{p}) \mathbf{q}} .
$$

The dynamic equation (25) is the discrete analogue to (20). The term including $\boldsymbol{\lambda}_{0}$ is merely an artefact of the discretization. However, it must be included in order to ensure proper conservation of energy.

\subsection{Integration algorithm}

The equations of motion (22) and (25) are conveniently solved simultaneously by means of Newton-Raphson iterations where the elements of the residual vector $\mathbf{r}=\left[\mathbf{r}_{q}^{T}, \mathbf{r}_{p}^{T}\right]^{T}$ are defined as

$$
\begin{aligned}
& \mathbf{r}_{q}=\Delta \mathbf{q}-\Delta t \mathbf{G}(\overline{\mathbf{q}})^{T} \mathbf{J}^{-1} \overline{\mathbf{G}(\mathbf{q}) \mathbf{p}} \\
& \mathbf{r}_{p}=\Delta \mathbf{p}+\Delta t \mathbf{G}(\overline{\mathbf{p}})^{T} \mathbf{J}^{-1} \overline{\mathbf{G}(\mathbf{p}) \mathbf{q}}+\Delta t\left[\frac{\partial V_{*}}{\partial \mathbf{q}^{T}}+\left(\frac{\partial \Phi_{*}}{\partial \mathbf{q}}\right)^{T} \boldsymbol{\lambda}-\mathbf{C}(\overline{\mathbf{q}})^{T} \boldsymbol{\lambda}\right]
\end{aligned}
$$


Table 1: Conservative time integration algorithm.
1) Initial conditions: $\mathbf{u}_{0}^{T}=\left[\mathbf{q}_{0}^{T}, \mathbf{p}_{0}^{T}\right]$
2) Prediction step:

$$
\mathbf{u}=\mathbf{u}_{n},
$$

3) Residual calculation:

$$
\mathbf{r}=\mathbf{r}(\mathbf{q}, \mathbf{p}) \text { from (27). }
$$

4) Update incremental rotation parameters:

$$
\begin{aligned}
& \mathbf{K}_{i j}=\partial \mathbf{r}_{i} / \partial \mathbf{u}_{j}, \\
& \delta \mathbf{u}=-\mathbf{K}^{-1} \mathbf{r} . \\
& \mathbf{u}=\mathbf{u}+\delta \mathbf{u}, \\
& \text { If } \left.\|\mathbf{r}\|>\varepsilon_{\mathbf{r}} \text { repeat from } 3\right) .
\end{aligned}
$$

5) Return to 2) for new time step, or stop.

The residual is reduced iteratively to zero by use of the linearized increment corresponding to changes in kinematics at $t_{n+1}$. This is performed in terms of $\delta \mathbf{u}=\left[\delta \mathbf{q}^{T}, \delta \mathbf{p}^{T}\right]^{T}$ via the equation

$$
\mathbf{K} \delta \mathbf{u}=-\mathbf{r},
$$

where the elements of the tangential stiffness matrix $\mathbf{K}$ follow from partial differentiation as

$$
\mathbf{K}_{i j}=\partial \mathbf{r}_{i} / \partial \mathbf{u}_{j} .
$$

The implementation of the algorithm is illustrated in pseudo-code form in Table 1.

\section{NUMERICAL EXAMPLES}

The accuracy and conservation properties are illustrated by two simple examples, - a freely rotating brick and steady precession of a Lagrangian top in a gravitational field.

\subsection{Free rotation of a rigid body}

First the properties of the homogeneous form of the algorithm, i.e. with $V(\mathbf{q})=0$, are considered by application to free rotation of a rigid body. The moment of inertia tensor with
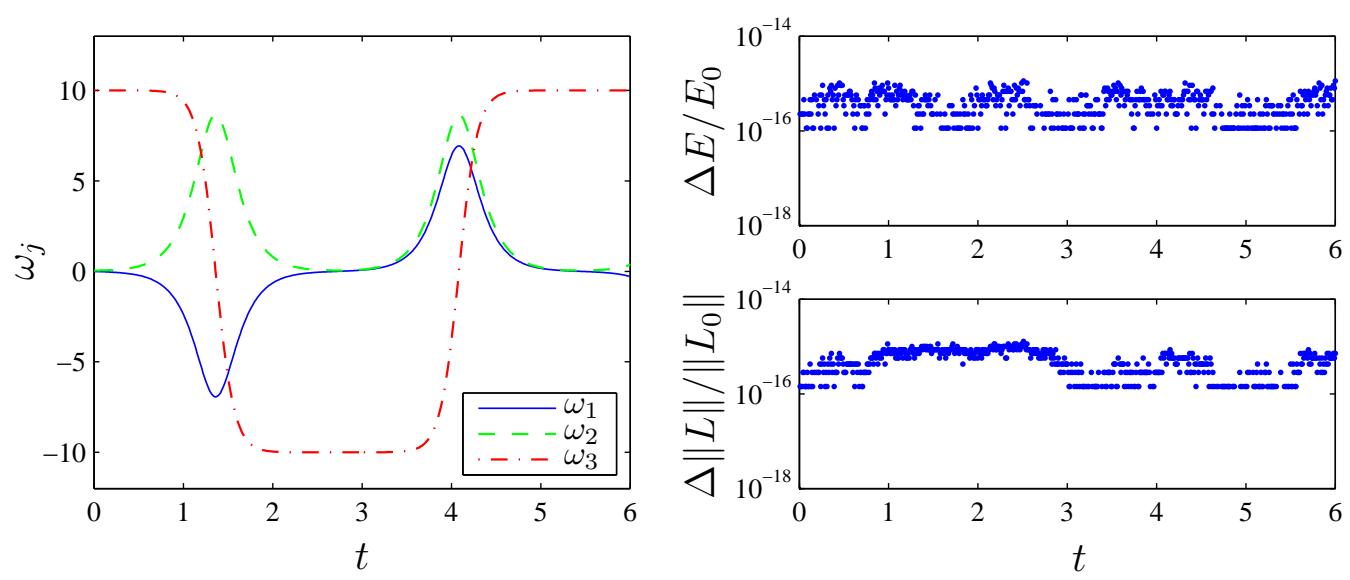

Figure 1: (a) Local angular velocity components, (b) Relative error on energy, (c) relative error on length of angular momentum vector. 
respect to the center of mass is chosen as $\mathbf{J}=\operatorname{diag}[13,5,10]$, which is equivalent to a box with side lengths $[1,3,2]$ and mass 12 . The motion is initiated by the initial angular velocity $\boldsymbol{\omega}_{0}=[0,0.05,10]^{T}$ leading to non-trivial rotation in which the body is reversed at regular intervals, see e.g. [3].

The unstable motion is illustrated in Fig 1 (a) in terms of the local angular velocity where the sign change in $\omega_{3}$ corresponds to the case where the box is turned upside down. The results are evaluated for the time step $\Delta t=0.01$ and an iteration tolerance of $\varepsilon_{\mathbf{r}}=10^{-8}$, which leads to conservation of the total mechanical energy $E$ and the length of the local angular velocity vector $\|\mathbf{L}\|$ within a relative error of $10^{-15}$ as illustrated in Fig. 1(b) and 1(c).

\subsection{Steady precession of top in gravitational field}

This example considering a Lagrangian top in a gravitational field is used to illustrate the properties of the algorithm when $V(\mathbf{q}) \neq 0$. The special case of steady precession without nutation is considered. The top is represented as a cone as illustrated in Fig. 2(a) with height $h$ and, max radius $r=h / 2$ and mass $m=\rho \pi r^{2} / 3$ with mass density is $\rho=2700$. The center of mass is located at a distance $l=3 h / 4$ from the tip, whereby the local moments of inertia become

$$
J_{1}=J_{2}=\frac{3}{80} m\left(4 r^{2}+h^{2}\right)+m l^{2}, \quad J_{3}=\frac{3}{10} m r^{2} .
$$

The top is located in a uniform gravitational field with acceleration $g=9.81$. In order to exhibit steady precession without nutation the following relation must be satisfied for the nutation angle $\theta$, the rate of precession $\dot{\varphi}$ and the spin velocity $\dot{\psi}$,

$$
\dot{\psi}=\frac{m g l}{J_{3} \dot{\varphi}}+\frac{J_{2}-J_{3}}{J_{3}} \dot{\varphi} \cos \left(\theta_{0}\right)
$$

see e.g. Goldstein [5]. The initial conditions correspond to the ones used in [2] and [3], $\dot{\varphi}=10$ and $\theta_{0}=\pi / 3$.
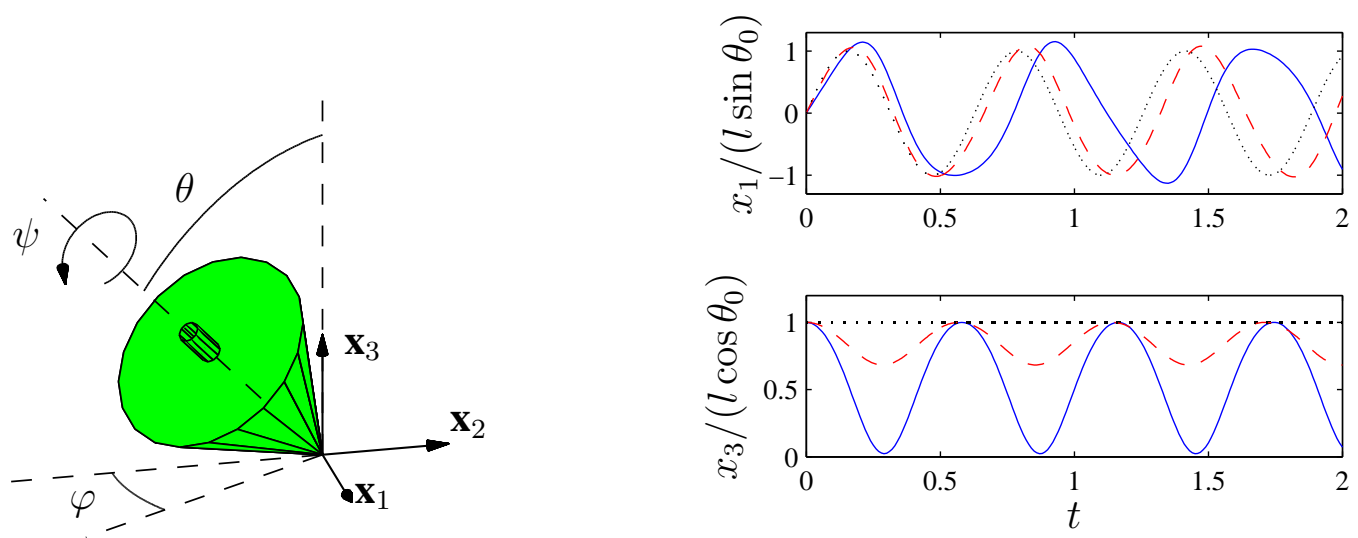

Figure 2: (a) Configuration of rotating top. (b) $x_{1}$-coordinate for center of mass. (c) $x_{3}$-coordinate for center of mass. $\Delta t=0.01(-), \Delta t=0.005(--)$, Analytical $(\cdots)$.

Simulations are performed for a time step of $\Delta t=0.01$ and an iteration tolerance of $\varepsilon_{\mathbf{r}}=$ $10^{-8}$. For these parameters the conserved quantities, namely the mechanical energy and the vertical component of the angular momentum, are conserved within an accuracy of $10^{-9}$. The $x_{1}$ and $x_{3}$-coordinates of the center of gravity are illustrated as the full line in Fig. 2(b) and 2 (c). The results show a significant period error compared to the analytical solution (dotted 
line), which leads to nutation since the criteria for steady precession is violated. This issue was discussed in [3]. However, when the time step of $\Delta t=0.005$ is used, the error is decreased by a factor of four as illustrated by the dashed line in Fig. 2(b) and 2(c), thereby illustrating the second order convergence of the present algorithm.

\section{CONCLUSIONS}

A conservative algorithm for rigid body rotation has been developed using a convected set of $3 \times 3$ orthonormal base vector components as generalized displacements. The equations of motion are derived from an augmented Hamiltonian where rigid body constraints equivalent to vanishing of all Green strain components are included via 6 Lagrange multipliers. However, in the present formulation it is illustrated that these can be eliminated by use of a a set of orthogonality condition between the generalized displacements and their conjugate momentum vector, leaving only a projection of the external potential gradient. A consistent time discretization scheme satisfying the conservation laws of energy and momentum is identified by considering a finite increment of the Hamiltonian. Furthermore, it is illustrated that when Lagrange multipliers are included in a conservative method, they serve a role as the effective reaction forces needed to uphold the constraints over a finite time interval, and thus they should be considered as interval bounded quantities rather than associated with the end interval points.

\section{REFERENCES}

[1] J. C. Simo, K. K. Wong, Unconditionally stable algorithms for rigid body dynamics that exactly preserve energy and momentum. International Journal for Numerical Methods in Engineering, 31, 19-52, 1991.

[2] P. Betsch, R. Siebert, Rigid body dynamics in terms of quaternions: Hamiltonian formulation and conserving numerical integration, International Journal for Numerical Methods in Engineering, 79, 444-473, 2009.

[3] M.B. Nielsen, S. Krenk, Conservative integration of rigid body motion by quaternion parameters with implicit constraints. International Journal for Numerical Methods in Engineering, 92, 734-752, 2012.

[4] P. Betsch, P. Steinmann, Constrained integration of rigid body dynamics. Computer Methods in Applied Mechanics and Engineering, 191, 467-488, 2001.

[5] H. Goldstein, C. Poole, J. Safko, Classical Mechanics, 3rd ed. Addison Wesley: San Francisco, 2001.

[6] O. Gonzalez, Exact energy and momentum conserving algorithms for general models in nonlinear elasticity. Computer Methods in Applied Mechanics and Engineering, 190, 1763-1783, 2000. 